\title{
Influence of Iron Core on Critical Current and Inductance of an HTS Double Pancake Coil
}

\author{
Meiying Yin ${ }^{1, a}$, Yong Zhao ${ }^{2}$, Zhuyong $\mathrm{Li}^{3}$, Yanling Hu${ }^{4}$, Ping Ruii ${ }^{4}$, Jing Zhang ${ }^{4}$, Ke Zhang ${ }^{4}$ and Fei $\mathrm{Xu}^{4}$ \\ ${ }^{1}$ Sino-Korean School of Multimedia Design, Shanghai University of Engineering Science, Shanghai, China \\ ${ }^{2}$ General Department of Power Grid Engineering Branch, China Energy Engineering Group Anhui Electric Power \\ Design Institute Company Ltd., Hefei, China \\ ${ }^{3}$ Department of Electrical Engineering, Shanghai Jiao Tong University, Shanghai, China \\ ${ }^{4}$ Dali Power Supply Bureau Repair Institute, Dali City, Yunnan, China
}

\begin{abstract}
In a high temperature superconducting (HTS) DC induction heater, an HTS coil and an iron core are essential to generate DC magnetic field and form the magnetic circuit. To understand effect of the iron core on the critical current and the inductance of the HTS coil, we prepared a double pancake coil (DPC) and an iron core in this study, and measured the critical current and inductance of the DPC. The experimental results were compared with the numerically calculated ones as well. The measured critical currents of the DPC without and with the iron core are 89 A and $84 \mathrm{~A}$, respectively. Thus, the iron core does affect the critical current. Moreover, the measured inductances are $6.94 \mathrm{mH}$ and $45 \mathrm{mH}$. The measured data of the critical currents and the inductances are in good agreement with the calculated results.
\end{abstract}

Keywords: Comsol; HTS coil; Inductance; Ic; Magnetic field.

\section{Introduction}

To ameliorate the poor efficiency of a traditional induction heater, a novel high temperature superconducting (HTS) DC induction heater has been suggested and being studied for commercial application [1]-[2]. A commercial industry-scale HTS DC induction heater with capacity of $1 \mathrm{MW}$ has been planned to be built in Shanghai Superconductor technology Co., Ltd. and Shanghai Jiao Tong University, China from 2014. For the HTS DC induction heater, an HTS coil is applied to generate DC magnetic field, and an iron core is necessary to form the magnetic circuit. Accordingly the critical current $\left(\mathrm{I}_{\mathrm{c}}\right)$ and the inductance of the HTS coil will be changed because of the iron core, which are the most important parameters of the HTS coil. Based on the critical current, the operating current of the coil is determined. And the inductance decides

\section{Experiment}

Fig. 1 shows a photograph of the insulated double pancake coil (DPC). As illustrated in Fig. 1, the DPC was wound on an anodizing aluminium bobbin; the bobbin was attached to a G10 panel; and the solder is used to connect both ends of the coil to current-leads. In the DPC,

\footnotetext{
a Corresponding author: seven840523@163.com
}

second generation (2G) HTS wire was insulated by Kapton tape [2].

The HTS wires in this paper are from SuNAM company, whose dimensions are $4.2 \mathrm{~mm}$ width and 0.25 $\mathrm{mm}$ thickness including a $20 \mu \mathrm{m}$ copper stabilizing layer in each side [3]-[4]. Besides, two brass tapes of $40 \mu \mathrm{m}$ thickness are reinforced on the wire, for improve mechanical properties. And Table I lists the specifications of the HTS wire and the DPC.

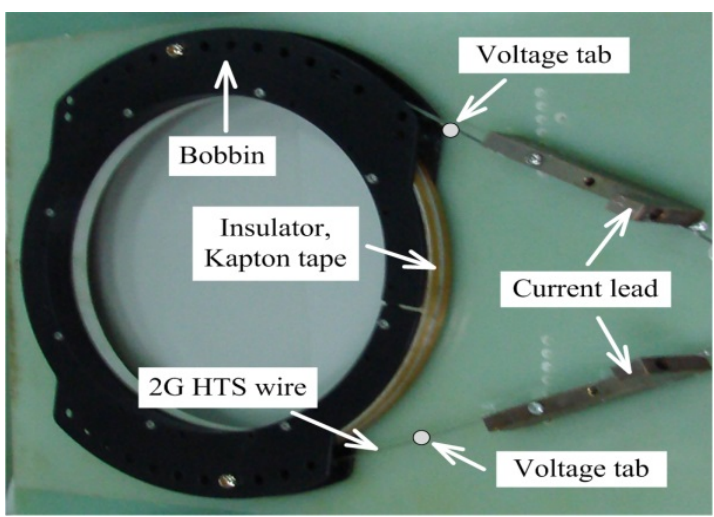

Fig.1 Photograph of the double pancake coil.

Fig. 2 indicates the photograph of the iron core mounting the double pancake coil in a liquid nitrogen bath. Considering the actual structure, the iron core is 
designed with twin air gaps [1]. The iron core and the $\mathrm{LN}_{2}$ bath are separable. Therefore, the double pancake coil and the bath will be separate from the iron core when the DPC is tested without the iron core.

Table 1. Specifications of the HTS wire and the DPC

\begin{tabular}{|c|c|}
\hline $\begin{array}{c}\text { 2G HTS wire } \\
\text { Width and thickness } \\
\text { Stabilizing layer } \\
\text { Lamination layer } \\
\text { Critical current, self-field } \\
\end{array}$ & $\begin{array}{c}4.2 \mathrm{~mm} \times 0.25 \mathrm{~mm} \\
\text { Copper, } 20 \mu \mathrm{m} \\
\text { Brass tape, } 40 \mu \mathrm{m} \\
170 \text { A@ } 97 \mathrm{~K}\end{array}$ \\
\hline $\begin{array}{c}\text { Double pancake coil } \\
\text { Bore size of bobbin } \\
\text { Inner and outer diameters of coil } \\
\text { Turns of DPC } \\
\text { Total length of wire } \\
\text { Width and thickness of insulator }\end{array}$ & $\begin{array}{c}230 \mathrm{~mm} \\
245 \mathrm{~mm}, 277 \mathrm{~mm} \\
58 \mathrm{turns} \times 2 \text { layers } \\
94 \mathrm{~m} \\
4.3 \mathrm{~mm} \times 0.025 \mathrm{~mm}\end{array}$ \\
\hline
\end{tabular}

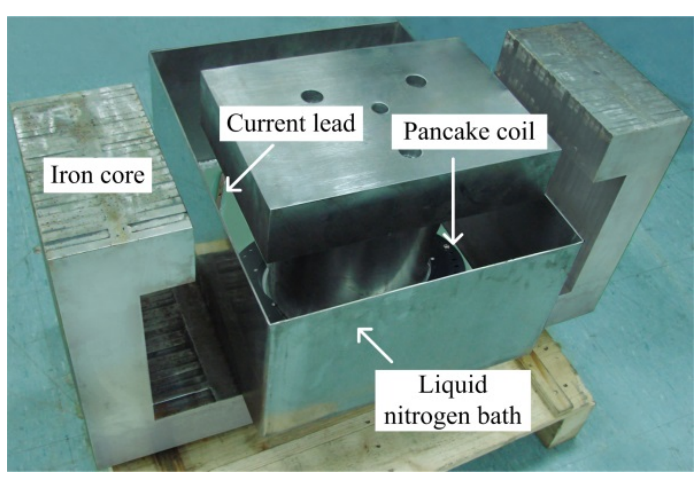

Fig.2 Photograph of the iron core, the double pancake coil, and the liquid nitrogen bath.

For the inductance evaluation of the DPC, the AC voltage and current are measured for the DPC. The AC voltage includes two parts: One is an inductive component from the inductance of the coil; the other is a loss component from the AC loss of the coil. Due to the $\mathrm{AC}$ loss is hysteresis loss and the loss voltage of the coil linearly depends on the frequency, the $\mathrm{AC}$ voltage and current are measured at a low frequency, $0.25 \mathrm{~Hz}$, to neglect the loss voltage and obtain the inductive voltage, Entire experiments are undergone at $\mathrm{LN}_{2}$ temperature of $77 \mathrm{~K}$.

\section{Numerical model}

In order to compare with the experimental data, a commercial finite element method (FEM) software, COMSOL Multiphysics, is utilized to analyse the critical current and the inductance of the DPC as numerical analysis. Fig. 3 illustrates the three-dimensional (3D) geometric model for the numerical analysis according to the actual dimensions. More detailed specifications of the iron core and the DPC are shown in Fig. 3 and the Table. The large quantity of mesh elements from the 3D geometry model will affect the calculated time. For improving the calculated speed, a quarter of the model is applied, and also the DPC is modelled as one coil. The material property of the iron core used in this analysis is referred to that in the material library of the COMSOL.

Generally, the critical current of superconducting coils is definitive by the one of the wire with the minimum critical current density [5]. This is because that once a point of the coil is quenched, the voltage of the whole coil will

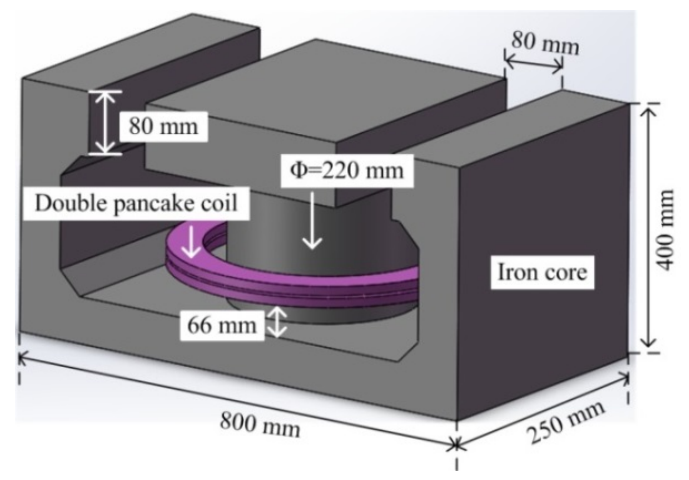

Fig.3 Schematic view of the geometric model about the iron core and the double pancake coil for numerical analysis.

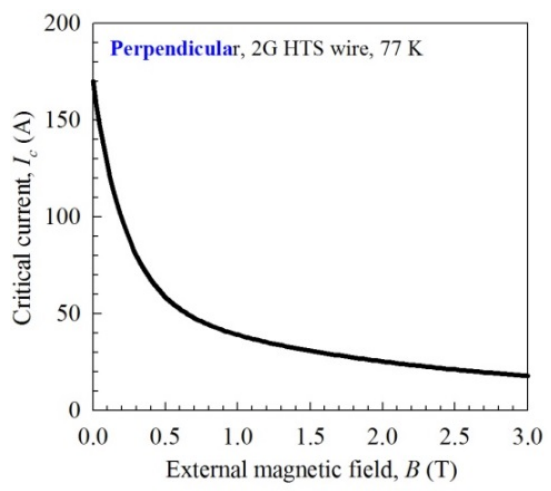

Fig.4 The external magnetic field dependence of the critical current for the 2 G HTS wire adopt in the numerical analysis.

increase to the critical value quickly due to the voltage of the quench point. The same method is applied to calculate the critical current of our DPC. As shown in Fig. 4, only the perpendicular component of the external magnetic field is considered in the critical current analysis. The data are provided from SuNAM company and can be expressed as:

$$
I_{c}=6.90+111 e^{-4.50 B}+52.0 e^{-0.519 B}
$$

where Ic denotes the critical current of the wire in the $\mathrm{DPC}$, and $\mathrm{B}$ denotes the perpendicular intensity of the external magnetic field.

The inductances of the double pancake coil can be calculated by using the Maxwell's equation and its auxiliary equation. The vector magnetic potential is analyzed from (2). Then, $\mathrm{B}, \mathrm{H}$ and $\mathrm{W}_{\mathrm{H}}$ are calculated by (3)-(4). Finally, the L can be calculated by (5).

$$
\begin{gathered}
\nabla \times(\nabla \times \vec{A})=\mu \vec{J} \\
\vec{B}=\nabla \times \vec{A} \\
W_{H}=\frac{1}{2} \int_{v} \vec{B} \cdot \vec{H} d v
\end{gathered}
$$




$$
L=\frac{2 W_{H}}{I^{2}}
$$

where $\mathrm{B}$ is the magnetic flux density, $\mathrm{H}$ means the magnetic field intensity, A denotes the vector magnetic potential, $\mathrm{J}$ is the current density, $\mathrm{u}$ is the permeability of the material, $I$ the current, $L$ the inductance and $\mathrm{W}_{\mathrm{H}}$ the magnetic field energy, respectively.

\section{Results and discussions}

\subsection{Critical current}

Fig. 5 shows the V-I characteristic curves of for the DPC with and without the iron core. As depicted in Fig. 5, the $\mathrm{I}_{\mathrm{c}}$ of the DPC without the iron core is $89 \mathrm{~A}$, and the one with the iron core is $84 \mathrm{~A}$ [2]. That is, the critical current is somewhat decreased for the existence of the iron core. Moreover, n-values are 42 and 18, respectively [6]. Thus, the n-value is greatly dependent on the iron core.

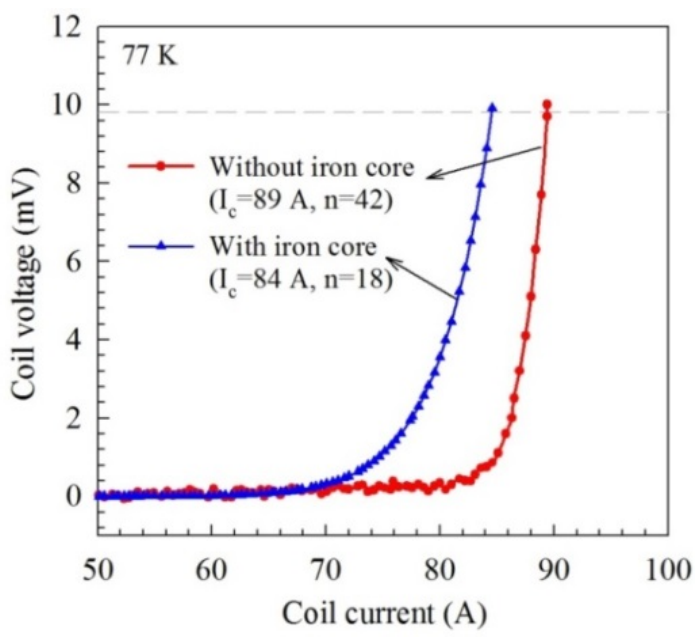

Fig.5 The data of V-I curve for the DPC with and without the iron core.

For elucidating the effect of the iron core on the magnetic field distribution. the magnetic field is calculated by the commercial program COMSOL. Furthermore, the $\mathrm{I}_{\mathrm{c}}$ of the DPC is analysed based on the data for the magnetic field distribution of the radial direction (x-axis). The radial direction of the DPC is perpendicular to the surface of the $2 \mathrm{G}$ HTS wire. Fig. 6 shows the calculated magnetic flux density distribution of the radial direction and the minimum critical current density for the cross section of the DPC without or with the iron core. A symbol, $\varphi$, means the direction of the current in the DPC.
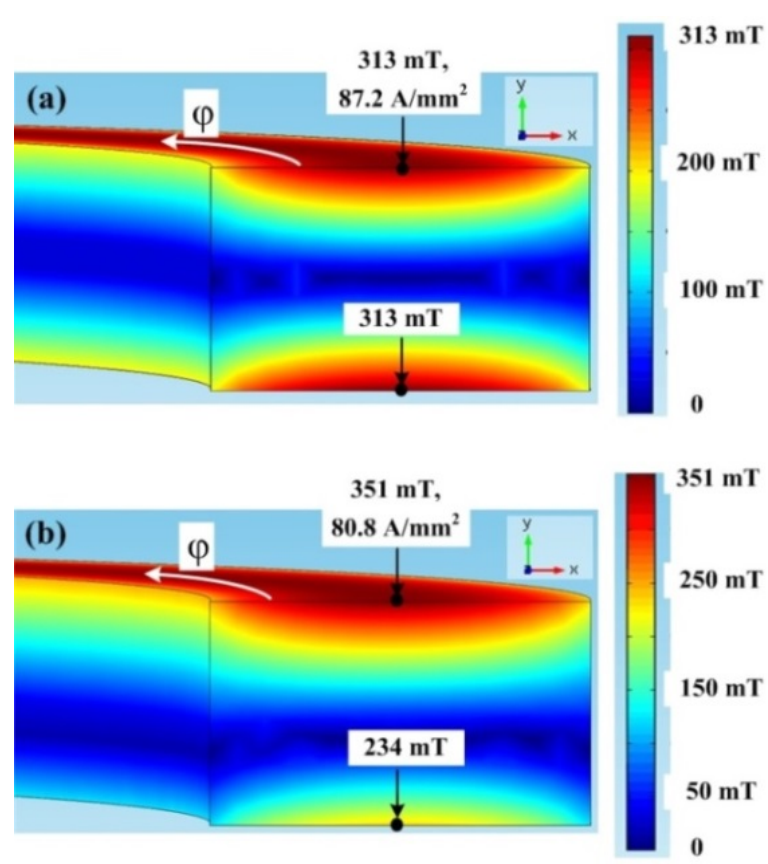

Fig.6 Calculated magnetic flux density distribution of the radial direction (x-axis) and the minimum critical current density.

As indicated in Fig. 6, the maximum magnetic flux densities are $313 \mathrm{mT}$ and $351 \mathrm{mT}$, respectively. The perpendicular intensity of the magnetic flux without the iron core is obviously smaller than that with the iron core. On the contrary, the minimum critical current density for the DPC without the core at the point of maximum magnetic densities, $87.2 \mathrm{~A} / \mathrm{mm}^{2}$, is larger than that with the iron core, $80.8 \mathrm{~A} / \mathrm{mm}^{2}$. According to the minimum critical current densities and the sectional area of the wire in Table I, we can obtain the critical currents, 91.7 A and 84.8 A, for the DPC without and with the iron core, respectively. These values are in good agreement with the measured ones of Fig. 5. Moreover, the critical current of the DPC will be changed according to different positions in the iron core.

\subsection{Inductance}

Fig. 7 shows the instantaneous voltage and current of the DPC. As shown in Fig. 7(a) and (b), it can be known that the phase between the coil voltage and the coil current is close to $90^{\circ}$, and the voltage leads the current. From the experimental data, the inductances of the DPC for both cases are obtained, which are $6.94 \mathrm{mH}$ and $45 \mathrm{mH}$, respectively [2]. This can be explained that the magnetic field energy becomes larger because of the iron core.

Fig. 8 presents the calculated magnetic field distribution of the coil with the iron core at the critical current, $84 \mathrm{~A}$. Through the magnetic field analysis, the inductance with the core is $42.4 \mathrm{mH}$, which is calculated from (2)-(5). Similarly, the calculated inductance without the iron core is $7.02 \mathrm{mH}$. These calculated values correspond well with the measured ones. 

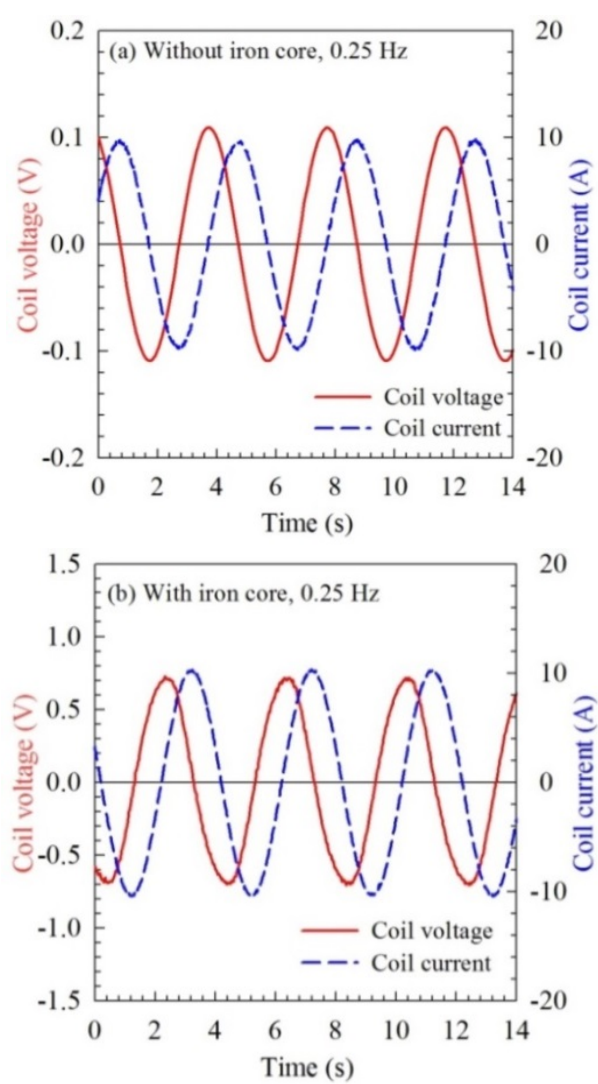

Fig.7 Instantaneous voltage and current of the DPC at frequency of $0.25 \mathrm{~Hz}$. (a) Without iron core: inductance, $\mathrm{L}=\mathrm{U} /(\mathrm{I} \times 2 \pi \times \mathrm{f})=0.109 \mathrm{~V} /(10 \mathrm{~A} \times 0.25 \mathrm{~Hz} \times 2 \pi)=6.94 \mathrm{mH}$, (b) with iron core: inductance, $\mathrm{L}=0.73 \mathrm{~V} /(10.4 \mathrm{~A} \times 0.25 \mathrm{~Hz} \times 2 \pi)=45 \mathrm{mH}$.

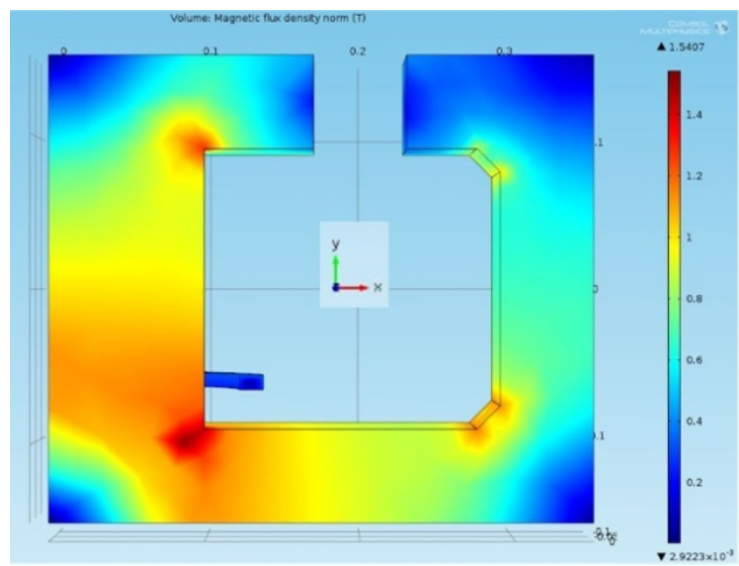

Fig.8 Calculated magnetic flux density distribution of the DPC with the iron core at the critical current.

\section{Conclusion}

In this study, we prepared a DPC and an iron core, to understand effect of the core on the critical current and the inductance. And then the critical current and the inductance were experimentally investigated for the DPC with or without the iron core. Moreover, the test results were discussed with numerical data.

The tested $\mathrm{I}_{\mathrm{c}}$ of the DPC without and with the core are $89 \mathrm{~A}$ and $84 \mathrm{~A}$, respectively. And the n-values are 42 and 18. Thus the $I_{c}$ and the $n$-value significantly denpend on the iron core. These experimental results correspond well with the simulated ones. For inductance results of the DPC with and without the core, the measured data are $6.94 \mathrm{mH}$ and $45 \mathrm{mH}$, respectively. Therefore, the discharging and charging time for the core will be much longer than that with no the iron core. The measured inductances also correspond well with the calculated ones.

\section{Acknowledgments}

This paper is supported by Training and Financing Plan for Young University and College Teachers in Shanghai, Project No.: ZZGCD15109.

\section{References}

1. M. Runde, N. Magnusson, C. Fulbier, and C. Buhrer, "Commercial induction heaters with hightemperature superconductor coils," IEEE Trans. Appl. Supercond., vol.21, no.3, pp.1379-1383, 2011.

2. Z. Y. Li, Y. Wang, J. Xu, D. Xu, Z. Hong, Z. Jin, K. Ryu, S. Yoon, and K. Cheon, "Design and Test Performance of $2 \mathrm{G}$ Pancake Coils for HTS DC Induction Heater Prototype," IEEE Trans. Appl. Supercond., vol.25, no.3, 4601205, 2015.

3. Superconductor, Nano \& Advanced Materials. [Online]. Available http://www.i-sunam.com/home/ en product, $1,3,1,1,1$.

4. S.-M. Choi, J.-W. Lee, S.-H. Moon, and S.-I. Yoo, "Microstructure analysis of $\mathrm{GdBa} 2 \mathrm{Cu} 3 \mathrm{O} 7-\delta$ coated conductors by the RCE-DR process," in MRS Proc., 2012, vol.1434, mrss12-1434-i05-06.

5. H. J. Kim, S. K. Baik, K. C. Seong, Zhuyong Li, YongHu Ma, and K. Ryu, "DC V-I Characteristics of a HTS tape for a $600 \mathrm{~kJ}$ SMES in an Oblique External Magnetic Field," Journal of Physics: Conference Series, vol.153, 012018, 2009.

6. H. B. Jin, Z. Y. Li, Y. H. Ma, K. Ryu, K. B. Park, and I. S. Oh, "Characteristics of a Bi-2212 Monofilar Superconducting Fault Current Limiting Element," IEEE Trans. Appl. Supercond., vol.19, no.3, pp.1847-1850, 2009. 